\title{
La sécurité des patients, une priorité pour la Confédération
}

\section{Oliver Peters}

Vice-directeur Office fédéral de la santé publique

\section{Les raisons d'agir}

En 1999, l'Institute of Medicine (USA) nous apprenait que, rien qu'aux Etats-Unis, entre 44000 et 98000 personnes décédaient chaque année en raison d'une erreur médicale [1]. La Task Force instituée début 2001 par le Département fédéral de l'intérieur estimait unanimement qu'en Suisse, le besoin d'amélioration en matière de sécurité des patients était comparable. Aujourd'hui encore, force nous est d'admettre, sur la base d'enquêtes régulières auprès des assurés [2], que les valeurs empiriques relevées à l'étranger sont applicables à la Suisse et qu'un patient sur dix environ subit un incident critique avec préjudice, qui serait évitable dans près de la moitié des cas. Le potentiel d'amélioration est donc important, et il faut s'y attaquer de façon systématique.

\section{Ce que la Confédération vise par son engagement}

La sécurité des patients est un élément clé dans la qualité des soins. Son amélioration constitue donc un élément central de la Stratégie fédérale en matière de qualité dans le système de santé (2009) et de la concrétisation de celle-ci (2011). La Confédération entend parvenir à réduire durablement le nombre d'incidents critiques évitables moyennant la garantie de traitements répondant à des critères de bonnes pratiques. L'art. 58 de la loi fédérale sur l'assurance-maladie (LAMal) lui offre la base légale requise.

\section{Programmes nationaux de qualité, instruments stratégiques essentiels}

La concrétisation de la stratégie fédérale mise principalement sur la réalisation de programmes nationaux de qualité. La fondation Sécurité des patients Suisse a sélectionné trois thèmes pour des projets pilotes dans ce domaine, dont la mise en œuvre est financée en grande partie par la Confédération: le programme pilote "progress! La sécurité en chirurgie» a pour objectif que les hôpitaux qui y prennent part appliquent systématiquement les normes existantes. Des inci- dents, tels qu'inversions de côté, erreurs d'identification, infections ou oubli de corps étrangers surviennent également en Suisse. L’application systématique d'une check-list chirurgicale permet de prévenir bon nombre de ces incidents indésirables. Le programme pilote vise le recours correct et systématique à la check-list entendue comme norme contraignante pour toute intervention invasive. Un deuxième programme pilote, "progress! La sécurité de la médication aux interfaces», élabore un concept d'intervention pour la Suisse qui permette d'éviter les erreurs de médication aux interfaces et d'augmenter la sécurité des patients. Neuf hôpitaux participent au projet d'approfondissement. Un premier programme régional d'amélioration de la sécurité de la médication a déjà été mené avec succès par la Fédération des hôpitaux vaudois. La troisième thématique abordée par la fondation Sécurité des patients Suisse, en coopération avec le groupe d'experts Swissnoso, est la diminution du nombre d'infections nosocomiales. Le troisième programme pilote «progress! La sécurité dans le sondage vésical» a pour but de réduire durablement le nombre d'infections dues à la pose de sondes vésicales.

\section{Coordination nationale et financement durable des activités favorisant la qualité}

Le Parlement a appelé le Conseil fédéral à renforcer ses activités dans le domaine de l'assurance qualité. Le Conseil fédéral entend donc accroître son engagement en la matière, comme il l'a d'ailleurs inscrit dans sa stratégie Santé2020, qui définit ses priorités pour la politique de la santé.

Aujourd'hui déjà, divers acteurs interviennent à différents niveaux dans le domaine de l'assurance qualité Mais ce qui fait défaut, c'est une coordination et une orientation stratégique: les efforts d'amélioration ne sont pas encore menés de façon suffisante et suffisamment systématique. Par un financement durable, le Conseil fédéral entend améliorer la coordination des activités déjà menées dans le domaine de l'assurance qualité, réduire le nombre d'incidents médicaux par des programmes concrets et augmenter la qualité des soins. La Confédération a donc l'intention d'étendre ses acti- 


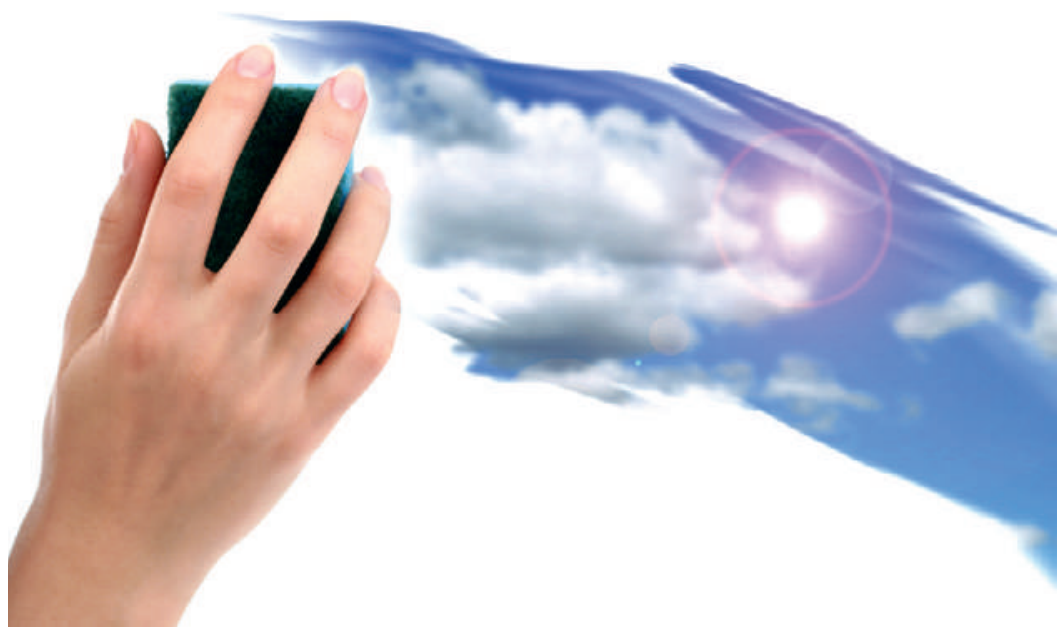

La transparence sur la qualité et la sécurité des soins est un préalable nécessaire pour une stratégie axée sur une amélioration constante.

vités au sein d'un réseau et de soutenir des projets et des programmes ayant pour but d'améliorer la qualité - et en même temps la sécurité des patients - dans l'assurance obligatoire des soins. Il s'agit notamment de recourir davantage aux programmes nationaux de qualité en s'appuyant sur l'expérience acquise en Suisse et à l'étranger. La fondation Sécurité des patients Suisse jouera là aussi un rôle de premier plan. Une contribution annuelle de quelque 3.50 francs par assuré ( 22 millions de francs en tout) est prévue pour financer ces différentes tâches.

\section{La transparence, condition clé d'une culture vivante de la sécurité}

La transparence sur la qualité et la sécurité des soins est le signe distinctif d'une culture ouverte de la qualité et de la sécurité, et donc un préalable nécessaire pour une stratégie axée sur une amélioration constante. L'Office fédéral de la santé publique (OFSP) publie depuis près de dix ans les "Indicateurs de qualité des hôpitaux suisses pour soins aigus» (www.bag.admin. ch/qiss): le premier de ces rapports portait sur l'année 2006. Cette publication vise la transparence sur la qualité des prestations fournies par les hôpitaux.

Depuis début 2009, l'art. 22a LAMal offre la base légale requise pour inclure tous les fournisseurs de prestations dans cette enquête. Développés en permanence, ces indicateurs se réfèrent aujourd'hui, en tout, à 48 tableaux cliniques et traitements tels qu'infarctus du myocarde, accident vasculaire cérébral, pneumonie ou encore pose de prothèses de la hanche ou du genou. De cette façon, $46 \%$ des cas d'hospitalisation dans les hôpitaux suisses pour soins aigus sont reflétés par un indicateur. Le nombre de cas publié de manière transparente permet de quantifier l'expérience qu'un hôpital a du traitement d'une maladie donnée. Le nombre de décès (taux de mortalité) sert de point de départ pour des analyses approfondies et de référence pour les améliorations à apporter dans les hôpitaux.

Active elle aussi dans le domaine de la mesure de la qualité, l'Association nationale pour le développement de la qualité dans les hôpitaux et les cliniques (ANQ) effectue des mesures de ce type depuis quelques années. Pour elle également, la publication transparente des résultats obtenus par hôpital constitue un objectif stratégique qu'elle réalise par étapes.

Mesurer la qualité n'est pas un but en soi, mais une condition essentielle pour identifier les déficits existants et lancer les processus d'optimisation nécessaires. La Confédération salue donc l'initiative prise par les hôpitaux de prendre les indicateurs de qualité de l'OFSP (en particulier les taux de mortalité) comme base pour mettre en place un processus d'évaluation par les pairs. Dérivé de celui de l'Initiative Qualitätsmedizin (IQM), ce processus innovera en étant interprofessionnel et en associant le secteur des soins. Cela permettra de boucler la boucle du cycle de qualité dans l'esprit d'une amélioration constante.

\section{Effets du processus d'optimisation}

Un processus d'optimisation structuré nous permettra d'abaisser le nombre d'incidents critiques évitables, réduisant par la même occasion les coûts dus à une mauvaise qualité. Sur la base d'une étude qu'il a menée, le professeur Charles Vincent estime que les patients qui subissent un incident critique avec préjudice (adverse event) passent en moyenne une semaine de plus que les autres à l'hôpital. Avec des incidents critiques estimés à $10 \%$ du nombre total de cas dont jusqu'à la moitié pourraient être évités, le potentiel d'économies est de plus d'un demi-milliard de francs suisses [3]. Chacun de ces incidents est lourd de conséquences pour les patients concernés. Il relève donc de la responsabilité éthique des acteurs de mettre ensemble en place un processus d'optimisation durable.

\section{Références}

1 Kohn et al. Institute of Medicine. 1999.

2 OBSAN Dossier 26. Erfahrungen der Allgemeinbevölkerung im Gesundheitssystem: Situation in der Schweiz und internationaler Vergleich (Perception du système de santé dans la population: situation en Suisse et comparaison internationale; publication en allemand, avec résumé en français).

3 Vincent C. L'essentiel sur la sécurité des patients. 2012. pp. 19 ss, qui se réfère à l'étude suivante: Vincent c et. al. Adverse events in British hospitals: preliminary retrospective record review. BMJ. 2001;322(7285):517-9. 